\title{
Microscopic Structure of the Metal-Insulator Transition in Two Dimensions
}

\author{
S. Ilani,* A. Yacoby, D. Mahalu, Hadas Shtrikman
}

\begin{abstract}
A single electron transistor is used as a local electrostatic probe to study the underlying spatial structure of the metal-insulator transition in two dimensions. The measurements show that as we approach the transition from the metallic side, a new phase emerges that consists of weakly coupled fragments of the two-dimensional system. These fragments consist of localized charge that coexists with the surrounding metallic phase. As the density is lowered into the insulating phase, the number of fragments increases on account of the disappearing metallic phase. The measurements reveal that the metal-insulator transition is a result of the microscopic restructuring that occurs in the system.
\end{abstract}

The ground state of electronic systems is found to change from metallic to insulating with increasing disorder. Disorder localizes the electronic wave functions and limits their ability to screen mutual Coulomb interactions. By reducing the density of carriers in such systems, both the effects of disorder and Coulomb interactions increase, thereby driving the system from metallic to insulating. Until recently, a metal-insulator transition (MIT) was believed to exist only in threedimensional systems, as only insulating behavior was predicted in lower dimensions (1). However, recent transport experiments have revealed the same phenomena in two-dimensional (2D) systems $(2,3)$. Despite extensive knowledge gained in recent experiments on the macroscopic characteristics of the 2D MIT phenomena, its microscopic properties remain unknown. We report on local compressibility measurements that unravel the microscopic structure that underlies the MIT in $2 \mathrm{D}$.

Compressibility reflects the ability of an electronic system to screen external charges. When an external charge is brought in close proximity to the surface of a metal, mobile electrons accumulate in its vicinity to screen it. A system that cannot redistribute its charge is, hence, incompressible. The electronic compressibility, therefore, provides a primary tool to understand the type of ground state that is formed by electrons. We used a single electron transistor (SET) to measure the local compressibility of a two-dimensional hole gas (2DHG) across the MIT. We use GaAs/ AlGaAs samples having a 2DHG formed 400 $\mathrm{nm}$ beneath the surface. An additional 400 $\mathrm{nm}$ of AlGaAs barrier material separates the

Braun Center for Submicron Research, Department of Condensed Matter Physics, Weizmann Institute of Science, Rehovot 76100, Israel.

*To whom correspondence should be addressed. Email: shahal.ilani@weizmann.ac.il
2DHG from a back gate, which enables us to tune the density of holes across the MIT (4) The 2DHG is accumulated solely by the back gate, and no intentional doping is present in the structure. The transport properties of these structures have been studied extensively, and a clear MIT phenomenology is observed (5). An aluminum SET positioned on the surface of the structure is used to measure the local chemical potential $(6-8), \mu$, as well as the local compressibility, $\kappa=n^{-2}[\delta n / \delta \mu]$ ( $n$ being the average density of holes), at dilution fridge temperatures $(50 \mathrm{mK})$.

Looking at the behavior of $\mu$ as a function of the hole density (Fig. 1A), it seems that starting from the metallic side, $\mu$ increases as the density is lowered - a behavior that corresponds to negative compressibility $(9,10)$. This behavior persists down to the critical density, $n_{\mathrm{c}}$, which is determined by transport measurements (11). When the density is further lowered, $\mu$ starts to decrease rapidly, indicating a positive and vanishing compressibility. As was described in previous works $(4,12)$, this change of sign of the compressibility constitutes the macroscopic thermodynamic signature of the MIT (13). However, in our previous structures (4), charge traps have limited our ability to determine the microscopic properties, and only the macroscopic thermodynamic behavior was determined. Here, we used a structure design in which the 2D carriers are accumulated using a gate (MOSFET principle) and not by intentional doping. This allows us to avoid the presence of charge traps and as a result enabled us to resolve the microscopic evolution of the MIT.

The microscopic behavior emerges as an intricate fine structure in the compressibility that is superimposed on the average macroscopic behavior. Two generic types of microscopic structures are apparent in the measured signal (Fig. 1). The larger structure is a staircase decrease of $\mu$ on the insulator side (arrows in Fig. 1A). However, a much finer structure appears when we directly measure the derivative of $\mu$ with respect to the backgate voltage, $\delta \mu / \delta V_{\mathrm{BG}}$ (Fig. 1B). This structure assumes the form of very sharp spikes that are superimposed on a smooth background. Because the back-gate voltage controls the average density, $\delta \mu / \delta V_{\mathrm{BG}}$ is a direct measure of the local inverse compressibility, $n^{2} \delta \mu / \delta n$. The emergent spikes in $\delta \mu / \delta V_{\mathrm{BG}}$ are the main focus of this work, as they correspond to microscopic processes that happen in the 2DHG near the MIT and their spectrum captures the fingerprint of these processes. These spikes commence already in the metallic phase, where a relatively sparse spectrum of negative spikes is observed (Fig. 1C). As the density is lowered across the transition, this spectrum becomes richer-both the number of spikes and their amplitudes increase substantially (Fig. 1D). Once deep in the insulating side, the spectrum is made up of many positive and negative overlapping spikes. The appearance of spikes superimposed on the continuous $\delta \mu / \delta V_{\mathrm{BG}}$ signal already hints at the presence of a new phase that develops as we approach the MIT.

A spike in the local compressibility signifies a discrete screening event. Such a process is rather unexpected if one assumes that the 2DHG can smoothly redistribute its charge. However, within various theories of the MIT, there are several fundamental processes that might break the smooth screening capability on the microscopic level. One set of theories $(1,14,15)$ suggests that strongly localized states must appear near the apparent MIT. Each such state can either be empty or hold a single electron. In this scenario, the sharp transition between the two situations will cause an abrupt jump in the local $\mu$ and a spike in $\delta \mu / \delta V_{\mathrm{BG}}$. Another set of theories (16, 17) claim that the MIT results from percolation in the electron liquid. In this scenario, puddles of charge are formed in the percolation landscape. The puddles can be charged only discretely - one electron at a timewhich results in having only a discrete set of back-gate voltages where the system is compressible. Because in both scenarios the system is incompressible between the discrete charging events, the spikes in $\delta \mu / \delta V_{\mathrm{BG}}$ are expected to be superimposed on an incompressible background $\left(\delta \mu / \delta V_{\mathrm{BG}}=1\right)$. This is not observed experimentally (Fig. 1C), where the observed background has very high compressibility $\left(\delta \mu / \delta V_{\mathrm{BG}} \ll 1\right)$. This suggests the coexistence of two phases: one with continuous response producing the compressible background and one with discrete response producing the spikes-namely, a two-liquid behavior. One can see (Fig. 1D) that as the system deepens into the insulating regime, the background $\delta \mu / \delta V_{\mathrm{BG}}$ increases toward the incompressible value $\left(\delta \mu / \delta V_{\mathrm{BG}}=1\right)$, whereas the number of spikes and their amplitudes increase. This behavior demonstrates that as 
the density of holes is reduced, the discrete phase grows at the expense of the coexisting compressible phase.

The above models are only suggested candidates for the nature of the discrete phase, and clearly, more complicated models, such as electron crystallization (18-24), should also be considered. However, the essential feature that all the possible descriptions would share is the local reorganization of charge, a necessary condition for the appearance of spikes. This locality implies that the onset of spikes must be accompanied by a spatial structure. We now demonstrate how this spatial structure is resolved experimentally even with a detector that is fixed at a single point. The idea is to use the SET not only as an electrometer but also as a top gate. In contrast to the back gate, which affects the density uniformly in space, the top gate influences the density in a manner proportional to the distance from its edge. A spike in Fig. 1 occurs when a charging event crosses the Fermi energy. This happens at a certain local density. Changing the back-gate voltage by $\delta V_{\mathrm{BG}}$ shifts this event from the Fermi energy. However, one can restore the local density and hence shift the event back to the Fermi energy by applying a compensating top-gate voltage $\delta V_{\mathrm{TG}}$. The further away the event is from the SET, the larger is $\delta V_{\mathrm{TG}}$ that is required in order to shift it back. Each charging event is, therefore, characterized by a "charging line" in the plane of $\left[V_{\mathrm{BG}}, V_{\mathrm{TG}}\right]$ (Fig. 2). The slope of the charging line, $s=$ $-\left(\delta V_{\mathrm{TG}} / \delta V_{\mathrm{BG}}\right)$, determines the position of the event in the 2DHG: An event happening just below the SET would have the smallest slope, $s_{\min }$. The further away the event is from the SET, the more vertical its slope will be (25).

Figure 2 shows how the overlapping spectrum of Fig. 1D disentangles into separate events, each having a different slope and amplitude. The span of slopes reflects the different positions of the events in the 2DHG. The smallest slope observed is $s_{\min }^{\exp }=2.26$, which corresponds to an event that occurs just under the SET (25). The largest slope observed, $s_{\min }^{\exp }=6.9$, corresponds to an event occurring $1.1 \mu \mathrm{m}$ away from the SET and defines the measurement horizon. This horizon is in good agreement with what is expected for a SET situated $400 \mathrm{~nm}$ above the 2DHG. A clear correlation can be seen between the position of an event and its amplitude - the closer it is to the SET, the stronger it appears. As the slopes can be measured rather accurately, we are able to position the events within the $2 \mathrm{DHG}$ with an accuracy of up to $\sim 20 \mathrm{~nm}$.

Our microscopic measurements, therefore, reveal that as the density of holes approaches the critical density from the metallic side, the $2 \mathrm{DHG}$ fragments into localized charge configurations that are distributed in space. The rather abrupt appearance of these events near $n_{\mathrm{c}}$ suggests that this fragmentation is the microscopic signature of the MIT. However, their simultaneous appearance in large numbers masks our ability to determine their size, mutual interactions, and the quan-

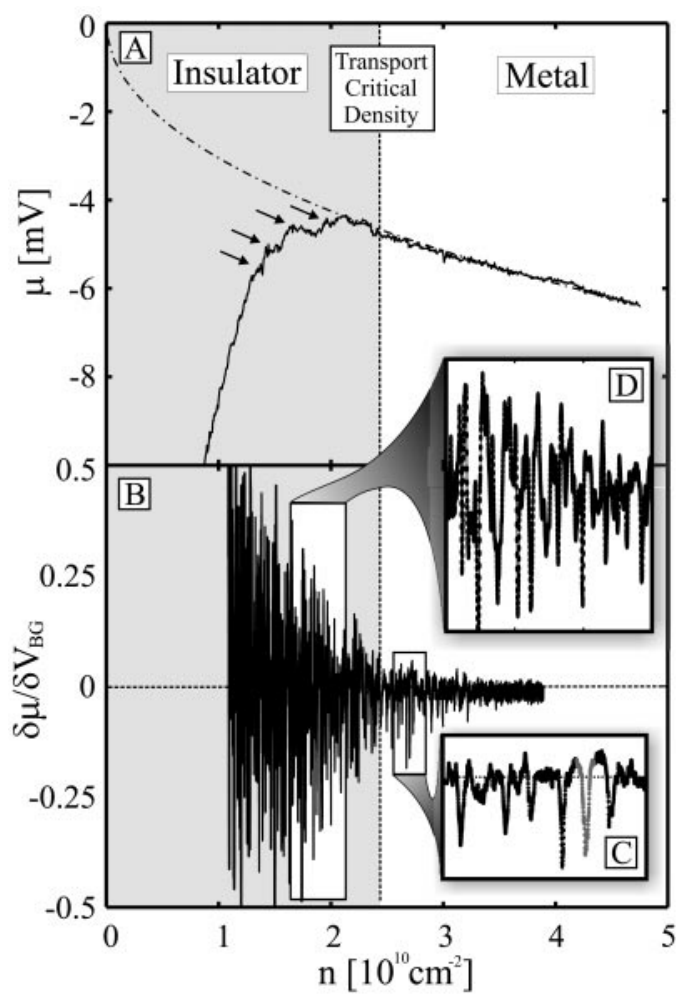

Fig. 1. (A) Density dependence of the local chemical potential, $\mu$, across the MIT. To measure this quantity, we deposited a metallic single electron transistor (SET) $400 \mathrm{~nm}$ above the 2DHG. By monitoring the current through the SET, we can deduce the local electrostatic potential, which directly determines the local $\mu$, given that the sample is in equilibrium (4). A transport measurement on the same device determines the MIT critical density, $n_{c}$ (dashed line). Above this density, $\mu(n)$ follows the prediction of the Hartree-Fock theory multiplied by 1.8 (dashed-dotted line). A rich reproducible microscopic structure is observed when we measure the derivative with respect to the back-gate voltage, $\delta \mu /$ $\delta V_{B G}$, using AC-lock-in techniques (B). This structure assumes the form of sharp spikes (a prototype spike is marked in gray), which emerge already on the metallic side (C) but become more dense and intense as the system crosses to the insulator side (D). Identical features of the spikes spectrum have been observed in measurements of several other devices. In each of these devices, a different spike spectrum was observed, being a fingerprint of the specific local disorder.

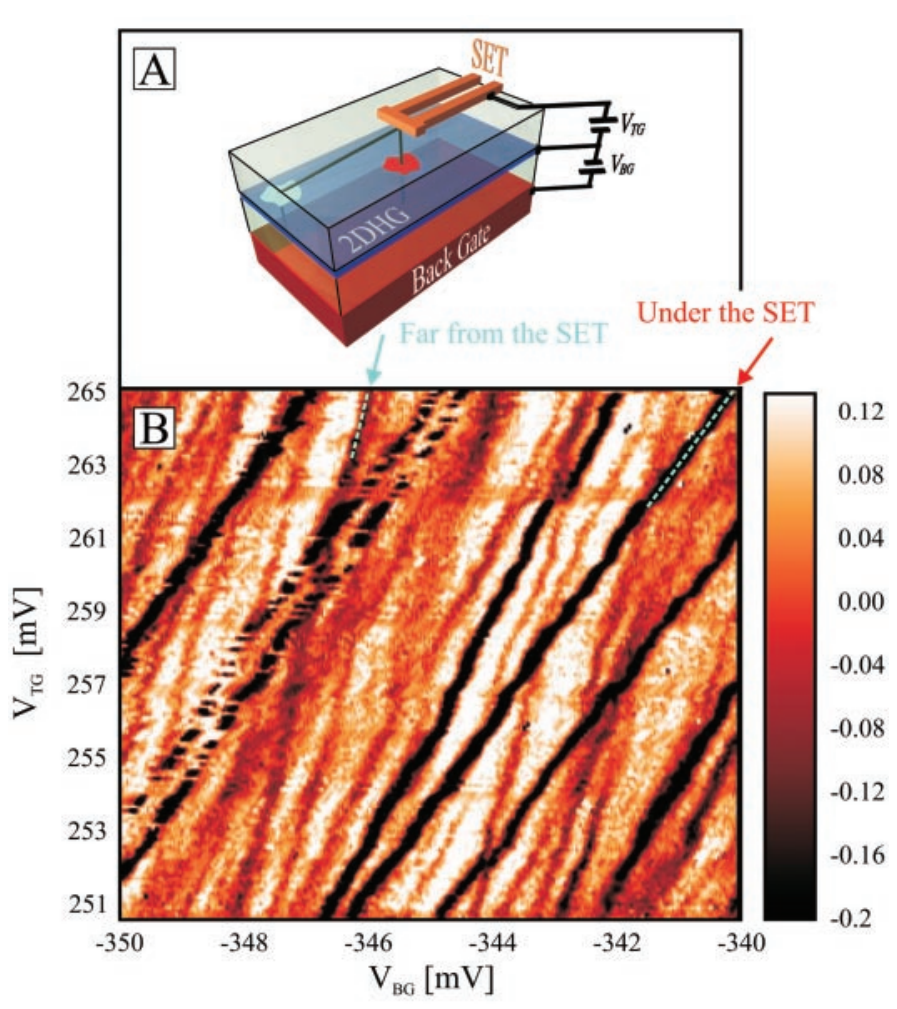

Fig. 2. (A) Scheme of the measurement circuit, which is used to identify the position of events within the 2DHG. (B) Color map representing the measured value of the derivative $\delta \mu / \delta V_{B G}$ as function of the backgate voltage, $V_{\mathrm{BG}}$, and top-gate voltage, $V_{T C}$. Each discrete event in the $2 \mathrm{DHG}$ gives rise to a line in this plot whose slope reflects the distance of the event from the SET (see text). Arrows mark the lines that correspond to two typical events, one that occurs directly under the SET and one that occurs far from the SET. 
under the SET at a constant value and generates a density gradient surrounding the SET. Because the spikes appear only at densities that are comparable to or smaller than $n_{\mathrm{c}}$, we can continuously reduce the area from which the spikes appear and hence their number.
The result of such a measurement is shown in Fig. 3. We plot our result in a coordinate system that is rotated to reflect the independent control over the density under the SET, $n_{\text {local }}$, and far away from the SET, $n_{\text {bulk }}$. In this plot, horizontal charging lines correspond to events that occur very far from
Fig. 3. Evolution of discrete events in response to an induced density gradient. Here, as in Fig. 2, the derivative $\delta \mu / \delta V_{B C}$ is measured as function of $V_{\mathrm{BG}}$ and $V_{\mathrm{TG}}$; however, now substantially higher voltages are applied so the density profile in the $2 \mathrm{DHG}$ is modified. The coordinate system is rotated with respect to Fig. 2: The horizontal axis corresponds to the density just below the SET, $n_{\text {local' }}$ and the vertical axis corresponds to the density far away from the SET, $n_{\text {bulk. }}$. In this coordinate system vertical (horizontal) charging lines correspond to local (distant) charging events. The circles mark avoided crossing between the charging lines that demonstrate the existence of tunneling amplitude between relatively distant charge configurations.

Fig. 4. Measured electrostatic interaction between events. This scan is similar to that in Fig. 3 but is done for local densities that are deeper in the insulator side. In this region, many encounters between charging lines assume a new form of crossing, which reflects the electrostatic interactions between events. The inset describes schematically a typical vertex. The colors of the charging lines (red/blue) associate them with the corresponding charging sites. The dashed lines labeled A, B, and C describe three different charging cycles of the two sites as function of the back-gate voltage.
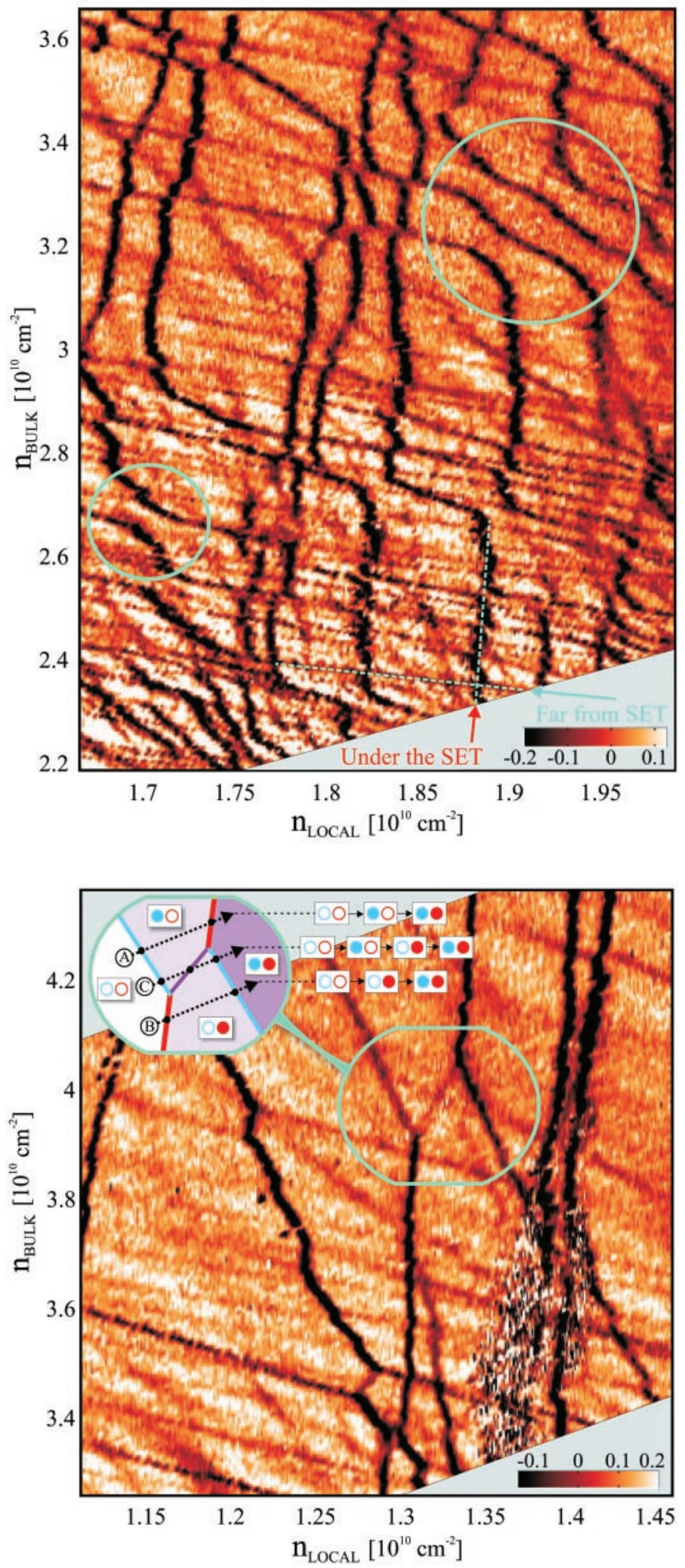

the SET, and vertical charging lines correspond to local events, occurring directly under the SET. As we go from the bottom of the figure to its top, we continuously evolve from a homogenous system, namely, $n_{\text {bulk }} \approx n_{\text {local }}$, to a system with a large density gradient, $n_{\text {bulk }} \gg n_{\text {local }}$. Noticeably, distant events (horizontal lines) are affected by $n_{\text {bulk }}$, and as this density exceeds $n_{c}$, most of them disappear rather abruptly, reflecting once more the fragmentation of the $2 \mathrm{D}$ system at the MIT. At the top of the scan, we are, therefore, left with only a few local charging events (vertical lines), which allow us to focus on the nature of the interactions between them. The coupling among the different charge configurations is apparent where the corresponding charging lines cross. Many encounters of avoided crossings are apparent, implying the existence of tunneling between the corresponding events. Encircled in this figure are large tunneling gaps that connect configurations whose spatial separation exceeds $1 \mu \mathrm{m}$. As only nearly overlapping configurations are mixed by tunneling (which is otherwise exponentially suppressed), this suggests that certain configurations extend more than $1 \mu \mathrm{m}$ in space - an order of magnitude more than the electron wavelength at this density.

As we go slightly deeper into the insulating side, the crossing of charging lines assumes a new form, which reflects the Coulomb interactions between events (Fig. 4). Here the slopes of the charging lines flip abruptly at two separate corners that are interconnected by a new charging segment. A typical vertex is drawn at the top of the figure. The red/blue charging lines correspond to charging of two events that are depicted by red and blue. Together with the new charging segment, they separate the four possible configurations of the two sites. The two sites can be charged with the use of the back gate in three different cycles, marked in Fig. 4 as A, B, and C. In cycle $\mathrm{A}$, the blue charging line is crossed before the red charging line, and, therefore, the blue site is charged before the red site. Because the red and blue charge configurations repel electrostatically, when the blue site is charged after the red site, as in cycle $\mathrm{B}$, its energy is pushed upward. This is manifested in the parallel shift of the blue line to higher back-gate voltages. Cycle $\mathrm{C}$ forms the most complicated charging sequence. It starts with the charging of the blue site, followed by an abrupt reorganization of the system in which the charge jumps from the blue site to the red site and finally a recharging of the blue site. The observed reorganization of charges signifies the importance of interactions in the ground state of the system near the MIT-a 
charging event will always be dressed by the reorganization of the system in nearby sites (26).

References and Notes

1. E. Abrahams, P. W. Anderson, D. C. Licciardello, T. V. Ramakrishnan, Phys. Rev. Lett. 42, 673 (1979).

2. S. V. Kravchenko, G. V. Kravchenko, J. E. Furneaux, V. M. Pudalov, M. D'lorio, Phys. Rev. B Cond. Matter 50, 8039 (1994).

3. For a comprehensive review of the MIT in $2 \mathrm{D}$, see $\mathrm{E}$. Abrahams, S. V. Kravchenko, M. P. Sarachik, Rev. Mod. Phys. 73, 251 (2001).

4. S. Ilani, A. Yacoby, D. Mahalu, H. Shtrikman, Phys. Rev. Lett. 84, 3133 (2000)

5. Y. Hanein et al., Phys. Rev. Lett. 80, 1288 (1998).

6. M. J. Yoo et al., Science 276, 579 (1997).

7. Y. Y. Wei, J. Weis, K. V. Klitzing, K. Eberl, Phys. Rev. Lett. 81, 1674 (1998).

8. N. B. Zhitenev et al., Nature 404, 473 (2000).

9. J. P. Eisenstein, L. N. Pfeiffer, K. W. West, Phys. Rev. Lett. 68, 674 (1992)

10. S. Shapira et al., Phys. Rev. Lett. 77, 3181 (1996).
11. The critical density was determined by simultaneous measurement on the same device, with the same technique as in (4)

12. S. C. Dultz, H. W. Jiang, Phys. Rev. Lett. 84, 4689 (2000)

13. A possible explanation of this behavior, which relates it to effects of disorder, has been given by $\mathrm{Si}$ and Varma [Q. Si, C. M. Varma, Phys. Rev. Lett. 81, 4951 (1998)].

14. B. L. Altshuler, D. L. Maslov, V. P. Pudalov, preprin available at xxx.lanl.gov/abs/cond-mat0003032.

15. A. L. Efros, B. I. Shklovskii, J. Phys. C Solid State Phys. 8, L49 (1975).

16. S. He, X. C. Xie, Phys. Rev. Lett. 80, 3324 (1998)

17. Y. Meir, Phys. Rev. Lett. 83, 3506 (1999).

18. An electron crystal, which is suggested by several theories (19-23) to appear at the MIT, might produce both continuos and discrete behaviors. Generally, the crystal deforms continuously to screen an external potential, producing a continuous response. However, occasionally, electrons collectively reorganize around pinning centers (24), causing an abrupt reduction of energy and hence a spike in $\delta \mu / \delta V_{B G}$. In this scenario, a single spike corresponds to a many-elec- tron charging event, rather than a single-electron charging, as in previous scenarios.

19. B. Tanatar, D. M. Ceperley, Phys. Rev. B 39, 5005 (1989).

20. S. T. Chui, B. Tanatar, Phys. Rev. Lett. 74, 458 (1995)

21. G. Benenti, W. Xavier, J. L. Pichard, Phys. Rev. Lett. 83 1826 (1999).

22. S. Chakravarty, S. Kivelson, C. Nayak, K. Voelker, Philos. Mag. B 79, 859 (1999).

23. J. Yoon, C. C. Li, D. Shahar, D. C. Tsui, M. Shayegan, Phys. Rev. Lett. 82, 1744 (1999).

24. I. M. Ruzin, S. Marianer, B. I. Shklovskii, Phys. Rev. B Cond. Matter 46, 3999 (1992).

25. The value of $s_{\min }$ could be estimated from the ratio of capacitances between the 2DHG and the top and back gates to be $s_{\min }=2.3$.

26. M. Pollak, M. Ortuno, in Electron-Electron Interactions In Disordered Systems, A. L. Efros, M. Pollak, Eds. (NorthHolland, Amsterdam, 1985), vol. 10, pp. 287-408.

27. We benefited from discussions with $M$. Brodsky, A. M. Finkel'stein, Y. Imry, Y. Meir, A. Stern, C. M. Varma, and N. B. Zhitenev. This work was supported by the MINERVA Foundation, Germany.

27 December 2000; accepted 4 April 2001

\section{Controlling Chemical Turbulence by Global Delayed Feedback: Pattern Formation in Catalytic CO Oxidation on Pt(110)}

\author{
Minseok Kim, Matthias Bertram, Michael Pollmann, \\ Alexander von Oertzen, Alexander S. Mikhailov, \\ Harm Hinrich Rotermund,* Gerhard Ertl
}

\begin{abstract}
Control of spatiotemporal chaos is one of the central problems of nonlinear dynamics. We report on suppression of chemical turbulence by global delayed feedback using, as an example, catalytic carbon monoxide oxidation on a platinum (110) single-crystal surface and carbon monoxide partial pressure as the controlled feedback variable. When feedback intensity was increased, spiralwave turbulence was transformed into new intermittent chaotic regimes with cascades of reproducing and annihilating local structures on the background of uniform oscillations. The global feedback further led to the development of cluster patterns and standing waves and to the stabilization of uniform oscillations. These findings are reproduced by theoretical simulations.
\end{abstract}

Control theory was originally developed for military and industrial applications, with the task of stabilizing missile trajectories or the course of technological processes $(1,2)$. Similar approaches are used today to steer elementary physical and chemical systems with chaotic dynamics. To control a chaotic system, its behavior is monitored and subsequent corrective perturbations needed to stabilize a chosen unstable orbit are computed. However, such exact methods (3) require substantial real-time computations and become impractical for the control of fast processes and large systems. An

Abteilung Physikalische Chemie, Fritz-Haber-Institut der Max-Planck-Gesellschaft, Faradayweg 4-6, 14195 Berlin, Germany.

*To whom correspondence should be addressed. Email: rotermun@fhi-berlin.mpg.de alternative is provided by empirical methods involving delayed feedback [see, e.g., (4)]. To realize such a feedback, a monitored variable is taken with a fixed time delay and used to generate a control signal that acts back on the system by changing one of its parameters. A feedback is global if the control signal represents a sum of contributions coming from many parts of the system. Various forms of delayed feedback have been discussed in optics, to suppress spatiotemporal instabilities in lasers (57). Further examples of global feedback control include suppression of plasma instabilities in gas discharges (8) and effects of charge transport in semiconductors $(9)$. Stabilization of unstable stationary states by delayed feedback in a stirred chemical reactor has also been experimentally demonstrated (10).

In addition to its control function, global feedback can also be used to modify spatiotemporal pattern formation and produce new kinds of patterns. In reactions of heterogeneous catalysis, some global feedback through the gas phase is intrinsically present and influences pattern formation (11-14). In experiments with artificial global nondelayed feedback in the photosensitive oscillatory Belousov-Zhabotinsky (BZ) reaction (15), formation of spatial clusters has been observed [similar patterns in this reaction are also found under external periodic forcing (16)].

Here we focus on experimental and theoretical investigations of the effect of global delayed feedback on chemical turbulence. In oscillatory reaction-diffusion systems, this form of turbulence results from the difference in diffusion constants of reactants. If this difference is sufficiently large, uniform kinetic oscillations may become unstable and spatiotemporal chaos sets in (17). In the developed turbulent state, both the amplitude and the phase of local concentration oscillations are strongly fluctuating. A characteristic property of such turbulence is spontaneous creation of multiple rotating spiral waves. This diffusion-induced turbulence is typical for oscillatory surface chemical reactions $(18,19)$ and has also been observed under special conditions in the BZ reaction $(20)$. On the basis of theoretical investigations of a general mathematical model, it was previously suggested $(21,22)$ that this form of turbulence can be suppressed by application of an appropriate global delayed feedback and that the feedback can also produce new kinds of spatiotemporal patterns. Here, we demonstrate the operation of global delayed feedback in an experiment with catalytic oxidation of $\mathrm{CO}$ on platinum.

In contrast to other oscillatory chemical reactions, the mechanism of $\mathrm{CO}$ oxidation on $\mathrm{Pt}(110)$ is relatively simple (19). Molecules of $\mathrm{CO}$ and $\mathrm{O}_{2}$ adsorb from the gas phase on the catalytic metal surface (the adsorption of $\mathrm{O}_{2}$ is 